\title{
Reliability Analysis of Multi-State Engine Units Utilizing Time-Domain Response Data
}

\author{
Yongfeng FANG \\ School of Electronic \& Mechanical Engineering, Guizhou University of Science Technology, Bijie \\ 551700, China \\ E-mail: fangyf_9707@126.com \\ Wenliang TAO \\ School of Electronic \& Mechanical Engineering, Guizhou University of Science Technology, Bijie \\ 551700, China \\ E-mail: twl@gzu.edu.cn \\ Kong Fah TEE \\ Department of Engineering Science, University of Greenwich, Kent ME44TB, United Kingdom \\ E-mail: K.F.Tee@gre.ac.uk
}

\begin{abstract}
A novel reliability-based approach has been developed for multi-state engine systems. Firstly, the output power of the engine is discretized and modeled as a discrete-state continuoustime Markov random process. Secondly, the multi-state Markov model is established. According to the observed data, the transition intensity is determined. Thirdly, the proposed method is extended to compute the forced outage rate and the expected engine capacity deficiency based on time response. The proposed method can therefore be used for forecasting and monitoring the reliability of the multi-state engine utilizing time-domain response data. It is illustrated that the proposed method is practicable, feasible and gives reasonable prediction which conforms to the engineering practice.
\end{abstract}

Keywords multi-state; engine system; Markov; reliability; time response

\section{Introduction}

One inherent weakness of classical reliability theory is that the system and the units are always described just as functioning or failed ${ }^{[1]}$. At any time, the system is in one of these two states. In the real world, many systems can perform their tasks at several different levels. A system that can have different task performance levels is named multi-state system $(\mathrm{MSS}){ }^{[2,3]}$. MSS reliability has received a substantial amount of attention in the past four decades and has been widely used and proven to be very beneficial to several industries such as power systems, engines, electronic products, etc ${ }^{[4,5]}$.

Received February 4, 2016, accepted April 3, 2016

Supported in part by the National Natural Science Foundation of China (61473331), the Foundation from the Excellent Researcher of Bijie University (G2013017, G2015003), the Science and Technology Foundation of Guizhou ([2014]2001), and the Project of Guizhou Province Experiment Demonstration Teaching Center 
The MSS was introduced in the 1970's ${ }^{[6,7]}$. In these works, the basic concepts of MSS reliability were formulated. Much work in the field of reliability analysis was devoted to the binary-state systems, where only the complete failures are considered ${ }^{[8,9]}$. Although multistate reliability models provide more realistic and more precise representations of engineering systems, they are much more complex and present major difficulties in system definition and performance evaluation. Currently, the multi-state reliability assessment instead of a two-state system assessment for the engine has been proposed ${ }^{[10-12]}$. However, the proposed methods for multi-state engine reliability assessment are not accurate especially when the engine unit reliability has marginal situation.

In recent years, the research study of Markov chain and semi-Markov chain has been increasing. The theory is gradually developed and its application in the reliability of the system has been explored ${ }^{[13-15]}$. System reliability, availability, average operating time can be predicted by using the Markov chains and semi-Markov Chain. The reliability of coal-fired generating units was studied by using a single Markov model ${ }^{[16,17]}$. The reliability problems of various engineering systems with discrete-state and continuous-time were presented and the problems were solved by using Markov model with discrete and continuous time ${ }^{[18-22]}$. However, there is limited literature on multi-state engine system reliability studied by using the Markov chains and semi-Markov chain. The reliability of multi-state generator has been studied by using discrete-state and continuous-time Markov model and the predicted results can be used for short-term forecasting of this type of equipment ${ }^{[23]}$.

On the basis of these articles, the multi-state random process of engine system has been studied by using discrete-state and continuous-time Markov model and semi-Markov model based on the actual failure of engine system. The appropriate multi-state Markov model has been established and the multi-state engine reliability based on time response can be assessed by using the proposed model. It is illustrated that the proposed method is practicable, feasible and gives reasonable prediction which conforms to the engineering practice.

\section{The Multi-State Markov Model}

At any time $t$ during the work period $T$ of an engine unit, the work capacity of the engine can be indicated by using real interval $[0, g]$, where $g$ is the maximum work capacity of the engine at time $t$. Clearly, this is continuous-state random process. However, the process can be substituted using discrete-state continuous-time process $G(t)$ which is elaborated as follows.

1) The two special states of the engine are denoted by 1 and $N$ which correspond to $g_{1}=0$ that the engine has completely failed and $g_{N}=g$ that the engine produces output energy at a normal level, respectively.

2) The interval $[0, g]$ can be divided into $N-2$ subintervals and the length of each subinterval is $\Delta g=\frac{g}{N-2}$.

3) If $G(t) \in((i-1) \Delta g,(i-1) \Delta g], i=2, \cdots, N-1$, the state of the random process $G(t)$ is denoted by $i(i=2,3, \cdots N-1)$ at time $t$ and its work energy is denoted by $g_{i}$.

4) The work energy $g_{i}$ of the random process $G(t)$ in state $i$ is the average energy of $((i-1) \Delta g,(i-1) \Delta g]$.

The original continuous-state random process $G(t)$ is converted to discrete-state continuous- 
time random process $G_{D}(t)$ through quantitative techniques. The random process $G_{D}(t)$ has $N(N=1,2, \cdots, N)$ different output energy levels $g_{i}$. The random process $G_{D}(t)$ can be described by using Markov stochastic process with its transition states. The transition of $N$ states to each other is illustrated in Figure 1. The transition from state $i$ to state $j$ is denoted by $a_{i, j}$ whereas the transition from state $N-1$ to state $N-2$ is denoted by $a_{N-1, N-2}$ and so on. Each state $i$ corresponds to engine work energy $g_{i}$. The $m$-th sojourn time in state $i$ of the unit is denoted by $T_{i}^{m}$. The time can be observed as a sample in unit service period and the whole sample can be denoted by $\left\{T_{i}^{(1)}, T_{i}^{(2)}, \cdots, T_{i}^{\left(k_{i}\right)}\right\}$. The number of unit for transition from state $i$ to state $j$ is denoted by $k_{i j}$ whereas the number of sojourn in state $i$ of the unit is denoted by $k_{i}$. The transition intensity $a_{i, j}$ of discrete-state continuous-time stochastic process can be determined as discussed in Section 3 .

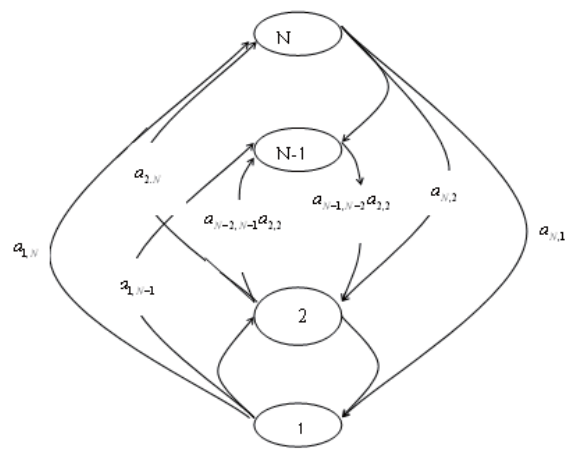

Figure 1 Multi-state Markov model for engine unit

\section{Determination of Transition Intensity}

$G_{D}(t)$ is a discrete-state continuous-time Markov process, if the transition time of the unit from state $i$ to state $j(i \neq j)$ can be neglected, only the instantaneous moment of the transition is interested, thus $G_{D}(t)$ can be considered as a discrete-state and discrete-time random process which is denoted by $G_{D i}(n), n=0,1, \cdots$. This is an embedded Markov process and this process can be entirely determined by using its initial state probability distribution and probability of one step transition which is denoted by $\pi_{i j}, i, j=1,2, \cdots, N$.

The cumulative probability distribution function of the unit transition from state $i$ to state $j(i \neq j)$ is shown as follows.

$$
F_{i j}(t)=1-e^{-a_{i j} t} .
$$

The first transition probability of the unit from state $i$ to state $j$ at time $t$ is denoted by $Q_{i j}(t), i, j=1,2, \cdots, N$. The nuclear matrix $\boldsymbol{Q}(t)$ of the random process $G_{D}(t)$ consists of all $Q_{i j}(t)$ and can be calculated as follows.

$$
Q_{i k}(t)=\int_{0}^{t}\left[1-F_{i 1}(u)\right] \cdots\left[1-F_{i k-1}(u)\right]\left[1-F_{i k+1}(u)\right] \cdots\left[1-F_{i k-1}(u)\right] \mathrm{d} F_{i k}(u) .
$$

Based on Equation (1), Equation (2) can be rewritten as follows.

$$
Q_{i k}(t)=\frac{a_{i k}}{\sum_{j=1}^{n} a_{i j}}\left[1-e^{-\sum_{j=1}^{N} a_{i j} t}\right] .
$$


The cumulative probability distribution function of sojourn time $T_{i}$ of the unit in state $i$ is written as follows.

$$
F_{i}(t)=\sum_{k=1}^{N} Q_{i k}(t)=1-e^{-\sum_{j=1}^{N} a_{i j} t} .
$$

It is shown from Equation (4) that $T_{i}$ is considered to obey the exponential distribution and the mean of $F_{i}(t)$ can be calculated as follows.

$$
T_{\text {imean }}=\frac{1}{\sum_{j=1}^{n} a_{i j}}=\frac{1}{A},
$$

where $A=\sum_{j=1}^{n} a_{i j}$.

On the other hand, the mean obtained by using observed samples is given as follows.

$$
\hat{T}_{\text {imean }}=\frac{\sum_{j=1}^{k_{i}} T_{i}^{(j)}}{k_{i}} .
$$

The total intensity of the transition from any states can be estimated by using Equation (7) which can be obtained by using Equations (5) and (6).

$$
\hat{A}=\frac{1}{\hat{T}_{\text {imean }}}=\frac{k_{i}}{\sum_{j=1}^{k_{i}} T_{i}^{(j)}} .
$$

The probability of one step transition can be obtained by using embedded Markov random processing.

$$
\pi_{i j}=\lim _{t \rightarrow \infty} Q_{i j}(t) .
$$

Equation (8) can then be further simplified by using Equation (4) as follows.

$$
\pi_{i j}=\frac{a_{i k}}{\sum_{j=1}^{N} a_{i j}} .
$$

The single transition intensity in state $i$ can be obtained by rearranging Equation (4) as follows.

$$
a_{i k}=\pi_{i j} \sum_{j=1}^{N} a_{i j} .
$$

The single step transition probability of embedded Markov chain can be computed by using unit work energy.

$$
\hat{\pi}_{i k}=\frac{k_{i k}}{k_{i}} .
$$

Finally, the transition intensity can be computed by using Equations (7), (10) and (11).

$$
\hat{a}_{i k}=\pi_{i k} \hat{A}=\frac{k_{i k}}{k_{i}} \frac{1}{\hat{T}_{\text {imean }}}=\frac{k_{i k}}{\sum_{j=1}^{k_{i}} T_{i}^{(j)}}, \quad i, k=1,2, \cdots, N .
$$

For the multi-state Markov system with $N$ states, the transition intensity can be written as follows.

$$
\sum_{j=1}^{N} a_{i j}=0
$$

where

$$
\hat{a}_{i i}=-\sum_{j=1, i \neq j}^{N} a_{i j} .
$$


In summary, the algorithm for determination of transition intensity for multi-state Markov system with $N$ states is given as follows.

Step 1 The system will be quantitatively processed by using the method described above. Every state $i$ of the engine will be corresponded to the output work energy $g_{i}$.

Step 2 The summation of sojourn time of the unit in every state $i$ can be computed by using the observed data.

$$
T_{\sum_{i}}=\sum_{m=1}^{k_{i}} T_{i}^{(m)}
$$

Step 3 The transition intensity from state $i$ to state $j$ is computed by using Equations (16) and (17).

$$
\begin{aligned}
& \hat{a}_{i j}=\frac{k_{i j}}{T_{\sum_{i}}}, \quad i \neq j . \\
& \hat{a}_{i i}=-\sum_{j=1, i \neq j}^{N} a_{i j} .
\end{aligned}
$$

\section{Worked Examples}

\subsection{Computation of Transition Intensity}

A fuel engine is used as an example to verify the proposed multi-state Markov model. The normal output power of the fuel engine is $288 \mathrm{KW}$ and its service period is limited to $T=5$ years. The multi-state Markov model can be established by using the above algorithm. The two special states of the engine correspond to $g_{1}=0 \mathrm{KW}$ that the engine has completely failed and $g_{4}=288 \mathrm{KW}$ that the engine produces output energy at a normal level. The interval $[0,288]$ can be divided into 2 subintervals and the length of each subinterval is

$$
\Delta g=\frac{288}{4-2}=144 \mathrm{KW} \text {. }
$$

Thus, the two intervals are given as $[0,144] \mathrm{KW}$ and $[144,288] \mathrm{KW}$. The other two output energy levels are computed as $g_{2}=123 \mathrm{KW}$ and $g_{3}=241 \mathrm{KW}$ by using the result that was observed in the last five years. The transition intensity $\hat{a}_{i j}$ from state $i$ to state $j$ is computed and shown as follows.

$$
\left[\begin{array}{llll}
-0.0933 & 0.0800 & 0.0133 & 0 \\
0.0294 & -0.3823 & 0.3235 & 0.0294 \\
0 & 0.0288 & -0.3846 & 0.3558 \\
0.0002 & 0.0001 & 0.0007 & 0.0010
\end{array}\right]
$$

The number of transition from state $i$ to state $j$ and the sojourn time in state $i$ of the unit is shown in Table 1. 
Table 1 The number of transition and the accumulated time

\begin{tabular}{ccccccc}
\multicolumn{6}{c}{ Table 1} & The number of transition and the accumulated time \\
\hline State & 1 & 2 & 3 & 4 & output energy $(\mathrm{KW})$ & accumulated time $T_{\sum_{i}}(\mathrm{~h})$ \\
\hline 1 & 0 & 6 & 1 & 0 & 0 & 75 \\
2 & 1 & 0 & 11 & 1 & 123 & 34 \\
3 & 0 & 3 & 0 & 37 & 241 & 104 \\
4 & 6 & 4 & 28 & 0 & 288 & 40711 \\
\hline
\end{tabular}

\subsection{Analysis of Four-State Model of Engine Unit}

The transition of four-state Markov model is shown in Figure 2. The steady-state probabilities of the states 1,2,3 and 4 are given as follows, respectively.

$$
P_{1}=0.0018, \quad P_{2}=0.0008, \quad P_{3}=0.0025, \quad P_{4}=0.9949 .
$$

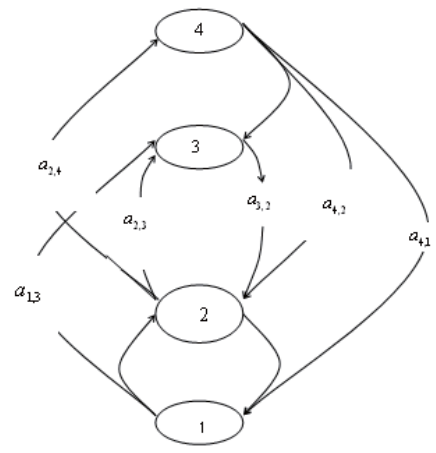

Figure 2 Four-state (MSS) Markov model for engine unit

The probabilities $P_{i}(t)(i=1,2,3,4)$ of the state $i$ can be obtained by solving the following differential equations (21) at any time under specific initial conditions.

$$
\begin{gathered}
\frac{\mathrm{d} P_{1}(t)}{\mathrm{d} t}=-\left(a_{12}+a_{13}+a_{14}\right) P_{1}(t)+a_{21} P_{2}(t)+a_{31} P_{3}(t)+a_{41} P_{4}(t), \\
\frac{\mathrm{d} P_{2}(t)}{\mathrm{d} t}=a_{12} P_{1}(t)-\left(a_{21}+a_{23}+a_{24}\right) P_{2}(t)+a_{32} P_{3}(t)+a_{42} P_{4}(t), \\
\frac{\mathrm{d} P_{3}(t)}{\mathrm{d} t}=a_{13} P_{1}(t)+a_{23} P_{2}(t)-\left(a_{31}+a_{32}+a_{34}\right) P_{3}(t)+a_{43} P_{4}(t), \\
\frac{\mathrm{d} P_{4}(t)}{\mathrm{d} t}=a_{14} P_{1}(t)+a_{12} P_{2}(t)+a_{13} P_{3}(t)-\left(a_{41}+a_{42}+a_{43}\right) P_{4}(t) .
\end{gathered}
$$

Once the probabilities of the states have been obtained, the stability probability of the engine in state $i$ can be calculated using Equation (22).

$$
p_{i}=\lim _{t \rightarrow \infty} P_{i}(t), \quad i=1,2,3,4 .
$$

\subsection{Reliability Prediction Based on Time Response}

In electrical engineering, forced outage rate (FOR) of engine is an important evaluation indicator. FOR is the probability that the engine will not be available for service when required and the output energy of the engine is 0 . It is a function of time which stops in state 1 as follows.

$$
\operatorname{FOR}(t)=P_{1}(t)
$$


FOR $(t)$ is computed based on initial conditions of the differential Equation (21). Four cases of initial conditions are studied and are preset as follows.

$$
\begin{aligned}
& \text { Case 1: } \quad P_{1}(0)=0, \quad P_{2}(0)=0, \quad P_{3}(0)=0, \quad P_{4}(4)=1, \\
& \text { Case 2: } \quad P_{1}(0)=0, \quad P_{2}(0)=0, \quad P_{3}(0)=1, \quad P_{4}(4)=0, \\
& \text { Case 3: } \quad P_{1}(0)=0, \quad P_{2}(0)=1, \quad P_{3}(0)=0, \quad P_{4}(4)=0, \\
& \text { Case } 4: \quad P_{1}(0)=1, \quad P_{2}(0)=0, \quad P_{3}(0)=0, \quad P_{4}(4)=0 .
\end{aligned}
$$

The calculated FOR $(t)$ for Cases 1, 2, 3 and 4 are shown in Figures 3, 4, 5 and 6, respectively. It is shown that the engine is stable after 80 hours and the stability probability in state 1 at that time is estimated as follows.

$$
p_{1}=\lim _{t \rightarrow \infty} P_{1}(t)=0.0018
$$

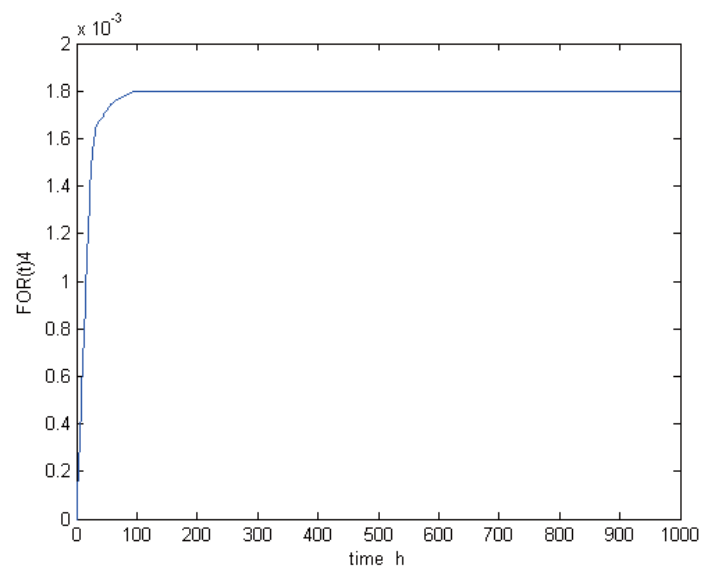

Figure $3 \mathrm{FOR}(t)$ under initial condition (24) for Case 1

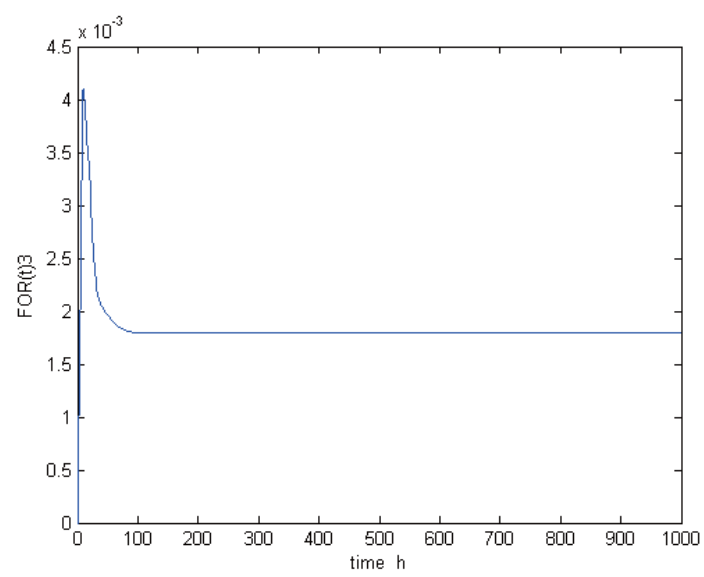

Figure $4 \mathrm{FOR}(t)$ under initial condition (25) for Case 2 


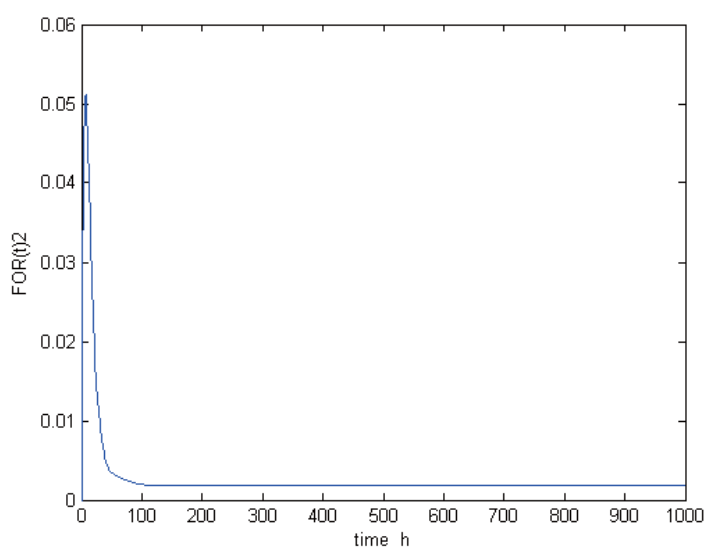

Figure $5 \operatorname{FOR}(t)$ under initial condition (26) for Case 3

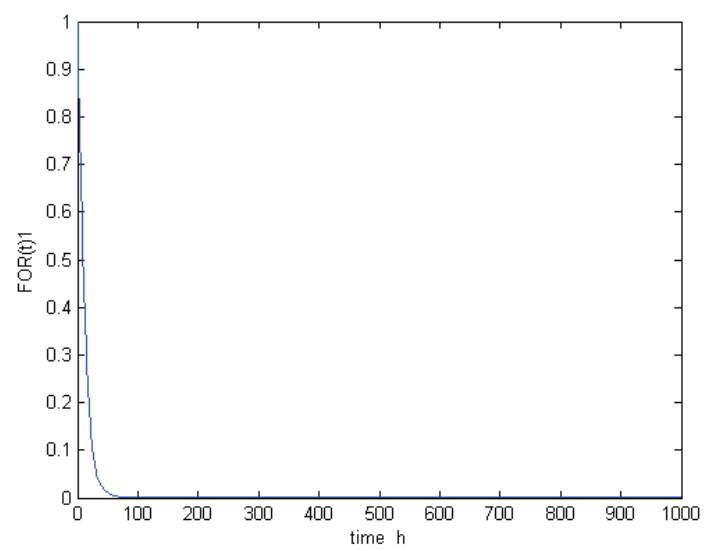

Figure $6 \mathrm{FOR}(t)$ under initial condition (27) for Case 4

Based on Equation (28), FOR of the engine is stabilized with the probability of 0.0018 under the initial conditions of (24), (25), (26) and (27). It is also noticed that the maximum FOR under the initial conditions of (25), (26) and (27) is larger than that under the initial condition of (24). The reason is that if state $i$ is closer to state 1 than state $j$ when the unit is transited from state $i$ to state $j$, the engine has a higher probability of malfunction. Obviously, the engine is turned into fault state when it is operated under the initial condition of (27) which can be validated by the real situation.

If the engine can be supplied to output capacity of $W=200 \mathrm{KW}$ at the 1000th hour, it will be transited to state 2 and the output energy can not be reached to $123 \mathrm{KW}$. In other words, the following capacity deficiency (CD) will be produced.

$$
\mathrm{CD} 2=(W-123)=76 \mathrm{KW} .
$$

If it will be transited to state 1 , the output energy can not be reached to the requirement. In other words, the following capacity deficiency (CD) will be produced.

$$
\mathrm{CD} 1=(W-0)=200 \mathrm{KW} \text {. }
$$


The expected capacity deficiency (ECD) is a function of time response and can be obtained as follows.

$$
\operatorname{ECD}(t)=P_{2}(t) \mathrm{CD} 2+P_{1}(t) \mathrm{CD} 1
$$

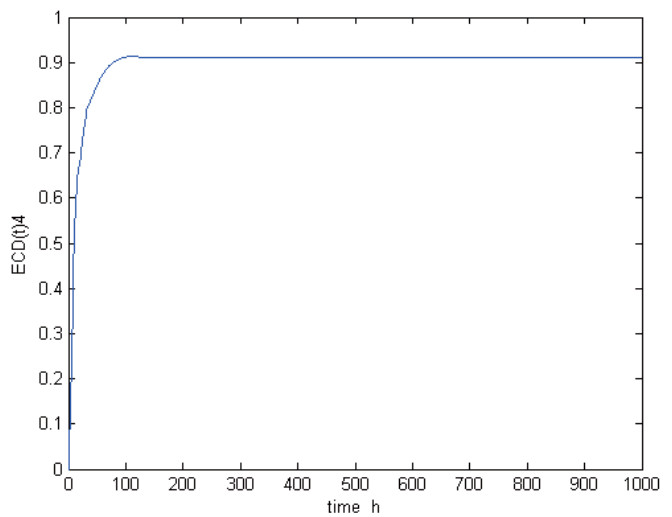

Figure $7 \mathrm{ECD}(t)$ under initial condition (24) for Case 1

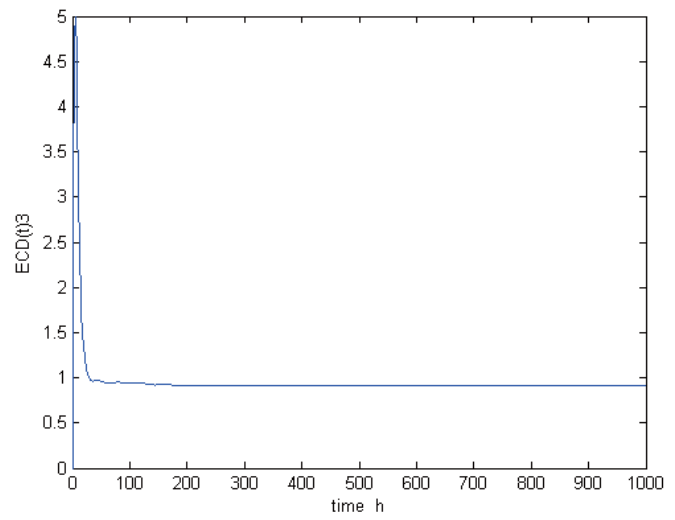

Figure $8 \mathrm{ECD}(t)$ under initial condition (25) for Case 2

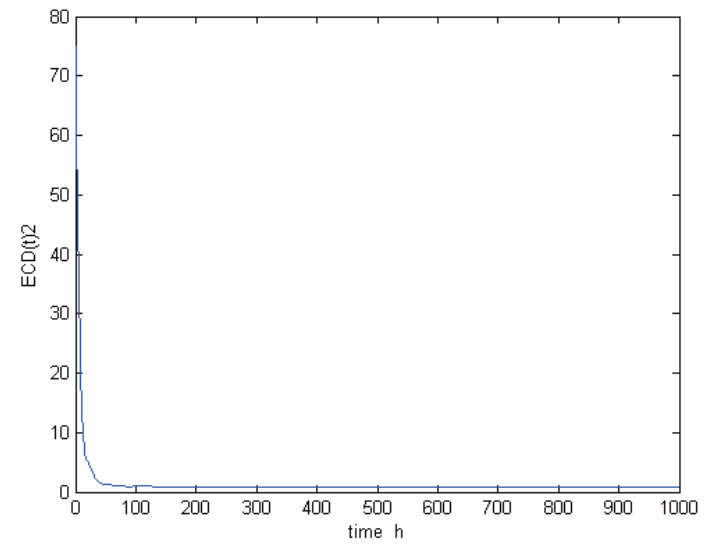

Figure $9 \operatorname{ECD}(t)$ under initial condition (26) for Case 3 


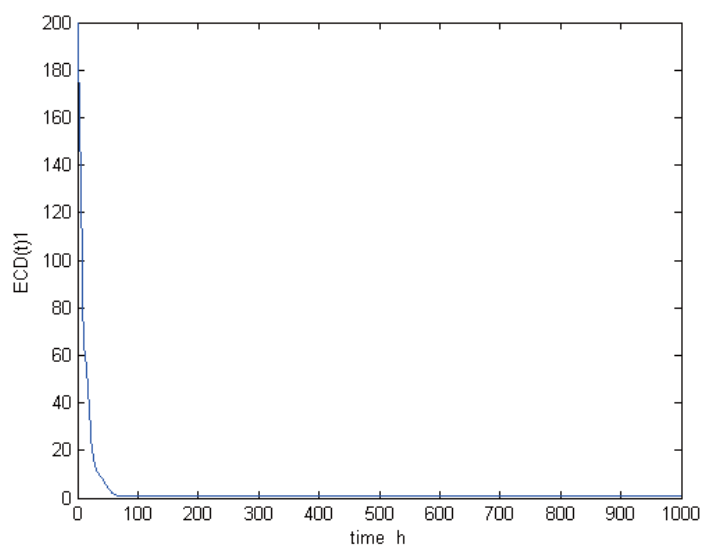

Figure $10 \operatorname{ECD}(t)$ under initial condition (27) for Case 4

The calculated ECD for Cases 1, 2, 3 and 4 under the initial conditions of (24), (25), (26) and (27) are shown in Figures 7, 8, 9 and 10. It is shown that the variation regular of the CD is the same as the variation regular of the FOR. Based on $\operatorname{ECD}(t) i$, the expected energy not supplied (EENS) at any time can be computed using Equation (32) as follows.

$$
\operatorname{EENS}(t) i=\int_{0}^{t} \operatorname{ECD}(u) i \mathrm{~d} u
$$

\section{Conclusions}

The output power of engine system is discretized and modeled as a discrete-state continuoustime Markov random process. The multi-state Markov model is then established. According to the observed data, the transition intensity is determined. The proposed method is then extended to compute the forced outage rate and the expected engine capacity deficiency with respect to time. The proposed method can be used for forecasting and monitoring the reliability of the multi-state engine utilizing time-domain response data. It is illustrated that the proposed method is practicable, feasible and gives reasonable prediction which conforms to the engineering practice.

\section{References}

[1] Fang Y, Chen J, Tee K F. Analysis of structural dynamic reliability based on the probability density evolution method. Structural Engineering and Mechanics, 2013, 45(2): 201-209.

[2] Natvig B. Multi-state systems reliability theory with application. New York: John Wiley \& Sons, 2011.

[3] Lisnianski A, Levitin G. Multi-state system reliability: Assessment, optimization, applications. World Scientific Press, 2003.

[4] Billinton R, Allan R N. Reliability evaluation of power systems. New York: Plenum Press, 1996.

[5] Manoukas G E, Athanatopoulou A M, Avramidis I E. Multimode pushover analysis based on energy equivalent SDOF systems. Structural Engineering and Mechanics, 2014, 51(4): 531-546.

[6] Barlow R E, Wu A S. Coherent system with multi-state components. Mathematics of Operations Research, 1978, 3(1): 275-281.

[7] Ross S. Multi-valued state component systems. The Annals of Probability, 1979, 7(2): 379-383.

[8] Fang Y, Wen L, Tee K F. Reliability analysis of repairable $k$-out- $n$ system from time response under several times stochastic shocks. Smart Structures and Systems, 2014, 14(4): 559-567. 
[9] Tee K F, Khan L R. Reliability analysis of underground pipelines with correlation between failure modes and random variables. Journal of Risk and Reliability, Proceedings of the Institution of Mechanical Engineers, Part O, 2014, 228(4): 362-370.

[10] Billinton R, Gao Y, Huang D, et al. Adequacy assessment of wind-integrated composite generation and transmission systems. Innovations in Power Systems Reliability, Springer Series in Reliability Engineering, London: Springer, 2011.

[11] Reshid M, Abd Majid M. A multi-state reliability model for gas fueled cogenerated power plant. Journal of Applied Science, 2011, 11(11): 1945-1951.

[12] Vosooq A K, Zahrai S M. Study of an innovative two-stage control system: Chevron knee bracing \& shear panel in series connection. Structural Engineering and Mechanics, 2013, 47(6): 881-898.

[13] Aven T. On performance measures for multi-state monotone systems. Reliability Engineering and System Safety, 1993, 41(3): 259-266.

[14] Brunelle R D, Kapur K C. Review and classification of reliability measures for multi-state and continuum models. Transactions of Institute of Industrial Engineers, 1999, 31(2): 1171-1181.

[15] Limnios N, Oprian G. Semi-Markov processes and reliability in statistics for industry and technology. Boston: Birkhauser, 2001.

[16] Goldner S. Markov model for a typical $360 \mathrm{MW}$ coal fired generation unit. Communication in Dependability and Quality Management, 2006, 9(1): 9-24.

[17] Jahanshahia M R, Rahgozar R. Free vibration analysis of combined system with variable cross section in tall buildings. Structural Engineering and Mechanics, 2012, 42(5): 715-728.

[18] Barbu V, Boussemart M, Limnios N. Discrete time semi-Markov processes for reliability and survival analysis. Communications in Statistic, Theory and Methods, 2004, 33(11): 2833-2868.

[19] Koroliuk V S, Limnios N, Samoilenko I. Poisson approximation of impulsive recurrent process with semiMarkov switching. J. Stochastic Analysis and Applications, 2011, 29(5): 769-778.

[20] Menshikova M, Petritis D. Explosion, implosion, and moments of passage times for continuous-time Markov chains: A semimartingale approach. Stochastic Processes and Their Applications, 2014, 124(7): 2388-2414.

[21] Barbu V, Limnios N. Semi-Markov chain and hidden semi-Markov models toward applications in lecture notes in statistic. Berlin: Springer, 2008.

[22] Janssen J, Manca R. Semi-Markov risk models for finance, insurance and reliability. Berlin, Germany: Springer-Verlag, 2007.

[23] Lisnianski A, Elmakias D, Laredo D, et al. A multi-state Markov model for a short-term reliability analysis of a power generating unit. Reliability Engineering and System Safety, 2012, 98(3): 1-6. 